\title{
Jian-Pi-Yi-Shen Formula Alleviates Chronic Kidney Disease in Two Rat Models by Modulating QPRT/NAD ${ }^{+} /$SIRT3/ Mitochondrial Dynamics Pathway
}

\author{
Xinhui Liu ${ }^{1},{ }^{1}$ Siqi Liu, ${ }^{1}$ Bing Zhang, ${ }^{1}$ Denggui Luo, ${ }^{1}$ Shiying Huang, ${ }^{2}$ Fochang Wang, \\ Lin Zheng, ${ }^{2}$ Jiandong Lu, ${ }^{1}$ Jianping Chen $\mathbb{C}^{2},{ }^{2}$ and Shunmin Li ${ }^{1}{ }^{1}$ \\ ${ }^{1}$ Department of Nephrology, Shenzhen Traditional Chinese Medicine Hospital, Guangzhou University of Chinese Medicine, \\ Shenzhen, Guangdong, China \\ ${ }^{2}$ Shenzhen Key Laboratory of Hospital Chinese Medicine Preparation, Shenzhen Traditional Chinese Medicine Hospital, \\ Guangzhou University of Chinese Medicine, Shenzhen, Guangdong, China
}

Correspondence should be addressed to Xinhui Liu; liuxinhui0317@163.com

Received 25 December 2020; Accepted 19 November 2021; Published 13 December 2021

Academic Editor: Adolfo Andrade-Cetto

Copyright (C) 2021 Xinhui Liu et al. This is an open access article distributed under the Creative Commons Attribution License, which permits unrestricted use, distribution, and reproduction in any medium, provided the original work is properly cited.

Objective. Jian-Pi-Yi-Shen formula (JPYSF) is a traditional Chinese herbal decoction and has been used for treating chronic kidney disease (CKD) in clinics for decades. However, the potential mechanisms have not been fully elucidated. This study was designed to test the efficacy of JPYSF in treating CKD and explore the underlying mechanism. Methods. Two CKD rat models were established by $5 / 6$ nephrectomy $(5 / 6 \mathrm{Nx})$ and feeding with adenine-containing feed, respectively. The intervention dose of JPYSF was $10.89 \mathrm{~g} / \mathrm{kg} / \mathrm{d}$ by gastric irrigation. Renal function was assessed by serum creatinine (Scr) and blood urea nitrogen (BUN). Periodic acid-Schiff (PAS) and Masson's trichrome staining were used to evaluate renal histopathological changes. The levels of nicotinamide adenine dinucleotide $\left(\mathrm{NAD}^{+}\right)$were measured by using the enzyme-linked immunosorbent assay kit. The proteins expressions of renal fibrosis, quinolinate phosphoribosyltransferase (QPRT), sirtuin 3 (SIRT3), and mitochondrial dynamics were determined and quantified by Western blot analysis. Results. The results show that administration of JPYSF significantly lowered Scr and BUN levels, improved renal tubular atrophy and interstitial fibrosis, and decreased renal extracellular matrix deposition in two CKD rat models. In addition, CKD rats exhibited suppressed QPRT/NAD ${ }^{+} / \mathrm{SIRT} 3$ signal, increased mitochondrial fission, and decreased mitochondrial fusion. JPYSF treatment promoted QPRT/NAD ${ }^{+} / \mathrm{SIRT} 3$ signal and restored mitochondrial fission/ fusion balance. Conclusion. In conclusion, administration of JPYSF effectively alleviated CKD progression in two rat models, which may be related with regulation of the QPRT/NAD ${ }^{+} /$SIRT3/mitochondrial dynamics pathway.

\section{Introduction}

The new data analysis suggested that in 2017, the global prevalence of chronic kidney disease (CKD) was $9.1 \%$ (697.5 million cases); CKD resulted in 1.2 million deaths and was the 12th leading cause of death worldwide $[1,2]$. Therefore, $\mathrm{CKD}$ is a global public health problem, and seeking effective treatments is urgent. Accumulating evidence indicates that traditional Chinese medicine (TCM) is effective in treating CKD, whether in CKD patients [3-5] or animal models [6-8]. Jian-Pi-Yi-Shen formula (JPYSF) has been used for treating $\mathrm{CKD}$ in clinics for decades. Our previous studies have found that JPYSF could protect the kidney from $5 / 6$ nephrectomy-induced CKD by regulating mitochondrial dynamics [9]; however, the underlying mechanism is unclear.

Mitochondria are dynamic organelles constantly undergoing fission and fusion events. Mitochondrial fusion is regulated by mitofusin (MFN) and optic atrophy 1 (OPA-1). The main regulator of mitochondrial fission is dynaminrelated protein 1 (Drp-1) [10]. Sirtuin 3 (SIRT3) is a nicotinamide adenine dinucleotide $\left(\mathrm{NAD}^{+}\right)$-dependent lysine deacetylase and is mainly located in the mitochondrial matrix and inner membrane [11]. Recent studies found that 
SIRT3 could inhibit mitochondrial fission and promote fusion in the treatment of acute kidney injury (AKI) [12], vitiligo [13], and Huntington's disease [14]. However, the role of SIRT3 in regulating mitochondrial dynamics in the CKD model remains unclear. Given that SIRT3 is regulated by levels of $\mathrm{NAD}^{+}$, it is necessary to further test $\mathrm{NAD}^{+}$status and upstream signal. Recently, de novo $\mathrm{NAD}^{+}$synthesis is considered to be important for health $[15,16]$. Poyan Mehr et al. showed that a bottleneck enzyme in de novo $\mathrm{NAD}^{+}$biosynthesis, quinolinate phosphoribosyltransferase (QPRT), defended renal $\mathrm{NAD}^{+}$and mediated resistance to AKI [17]. We asked the question of whether the QPRT/ $\mathrm{NAD}^{+} /$SIRT3/mitochondrial dynamics pathway was impaired in CKD and could be regulated by JPYSF treatment. In the present study, we established two CKD rat models by $5 / 6$ nephrectomy $(5 / 6 \mathrm{Nx})$ and feeding with adenine-containing feed, respectively. The efficacy of JPYSF was first determined, and QPRT/NAD ${ }^{+} /$SIRT3/mitochondrial dynamics pathway was subsequently measured.

\section{Materials and Methods}

2.1. Preparation of JPYSF Extract. JPYSF is composed of eight traditional Chinese herbal medicines, including Astragalus mongholicus Bunge (Fabaceae), Atractylodes macrocephala Koidz. (Asteraceae), Dioscorea oppositifolia L. (Dioscoreaceae), Cistanche deserticola Ma (Orobanchaceae), Wurfbainia vera (Blackw.) Skornick. and A. D. Poulsen (Zingiberaceae), Salvia miltiorrhiza Bunge (Lamiaceae), Rheum palmatum L. (Polygonaceae), and Glycyrrhiza uralensis Fisch. ex DC. (Fabaceae). The plant names have been validated with https://mpns.science.kew.org/ mpns-portal/. All raw herbs were weighed and boiled

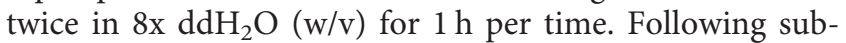
sequent centrifugation, the supernatant was dried by a freeze dryer and stored at $-80^{\circ} \mathrm{C}$. The chemical profile and quality control of JPYSF extract have been well described by using ultraperformance liquid chromatography with quadrupole time-of-flight tandem mass spectrometry (UPLC-Q-TOFMS/MS) in our previous study [18]. Briefly, a total of 71 compounds were identified from JPYSF extract, including saponins, flavonoids, sesquiterpenoids, coumarins, phenylpropanoids, anthranones, anthraquinones, tannins, phenolic acids, and others. Twelve main constituents were chosen as chemical markers to evaluate the quality of JPYSF extract. The minimum quantities (in $\mu \mathrm{g} / \mathrm{g}$ ) of these 12 constituents present in JPYSF extract dry powder are 1361.01 for rhein, 519.60 for salvianolic acid A, 251.73 for liquiritin, 98.03 for acteoside, 76.06 for calycosin 7-O-glucoside, 61.64 for rosmarinic acid, 41.39 for formononetin, 25.03 for calycosin, 13.05 for astragaloside IV, 8.95 for atractylenolide I, 3.45 for dioscin, and 0.21 for tanshinone IIA. This standard guarantees the quality of JPYSF extract used in our study.

2.2. Animals. All animal experiments were conducted with protocols approved by the Experimental Animal Ethics Committee of Guangzhou University of Chinese Medicine (No. 20190226020). Healthy male Sprague-Dawley (SD) rats weighted 150-180g were purchased from Guangdong Medical Laboratory Animal Center (Foshan, China). After one week of acclimatization, all rats were randomly assigned to two independent experimental designs. In experiment I, the rats were randomly divided into the following groups ( $n=6$ rats per group): (1) sham group; (2) 5/6 nephrectomyinduced CKD group (5/6 Nx-CKD); (3) JPYSF treatment group (CKD + JPYSF). The 5/6 Nx operation was performed in accordance with our previous publication [9]. The sham group only exposed the kidney without destroying the kidney tissue. After 12 weeks of 5/6 Nx operation, JPYSF extract was administered to CKD rats at the dose of $10.89 \mathrm{~g} /$ $\mathrm{kg} / \mathrm{d}$ for 12 weeks by gastric irrigation. In experiment II, the rats were randomly divided into the following groups $(n=6$ rats per group): (1) control group; (2) adenine-induced CKD group (adenine-CKD); (3) JPYSF treatment group (CKD + JPYSF). Adenine-induced CKD was performed by feeding adenine (Sigma-Aldrich, St Louis, MO, USA) in feed at a concentration of $0.75 \% w / w$ for 4 weeks $[8,19]$. Rats in the CKD + JPYSF group began to receive JPYSF treatment $(10.89 \mathrm{~g} / \mathrm{kg} / \mathrm{d}$, orally) from the third week for 4 weeks. At the end of experiments, all rats were anesthetized, and blood samples were obtained from abdominal aorta. The rats were euthanized by cervical dislocation without regaining consciousness. Kidneys were rapidly harvested and processed for further analysis. The animal experiment design is shown in Figure 1.

2.3. Serum Biochemistry. Serum samples were obtained by centrifuging blood for $10 \mathrm{~min}$ at $2,000 \mathrm{rpm}$ at $4^{\circ} \mathrm{C}$. The levels of serum creatinine (Scr) and blood urea nitrogen (BUN) were measured by a creatinine serum detection kit and a BUN detection kit (StressMarq Biosciences, British Columbia, Canada), respectively, following the manufacturer's instructions.

2.4. Histological Analysis. The paraffin-embedded kidney sections were performed periodic acid-Schiff (PAS) staining and Masson's trichrome staining to evaluate renal pathological injury and tubulointerstitial fibrosis. All images were captured by using the Nikon microscope and NIS-Elements BR software version 4.10 .00 (Nikon, Japan).

2.5. Western Blotting. The kidney cortexes were homogenized in RIPA buffer (Cell Signaling Technology, Beverly, MA, USA) containing a protease inhibitor cocktail. Equal amounts of kidney cortex lysates were loaded on and electrophoresed through $7 \%$ or $10 \%$ SDS-PAGE gels and were then transferred to nitrocellulose membranes (Millipore, Billerica, MA, USA). After blocking with 5\% skim milk, the membranes were incubated with primary antibodies against fibronectin (FN, 1:250), type IV collagen (Col-IV, 1:250) (Abcam, Cambridge, MA, USA), $\alpha$-smooth muscle actin $(\alpha$-SMA, $1: 1000)$, QPRT $(1: 500)$, $\beta$-actin (1:5000) (Sigma-Aldrich, St Louis, MO, USA), sirtuin 3 (SIRT3, 1:1000) (Proteintech, Wuhan, China), dynamin-related protein 1 (Drp-1, $1: 1000)$ (Cell Signaling 


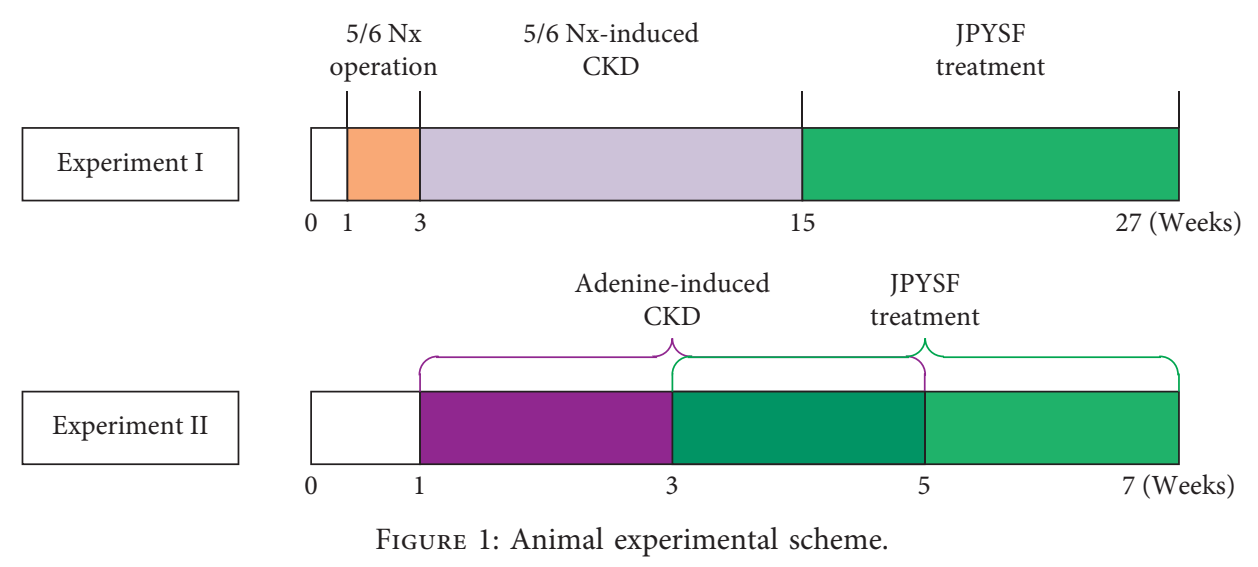

Technology, Beverly, MA, USA), and optic atrophy 1 (OPA-1, 1:2000) (BD Biosciences, San Jose, CA, USA) at $4^{\circ} \mathrm{C}$ overnight. After incubation with horseradish peroxidase (HRP)-conjugated secondary antibodies (1:2000) (Life Technologies, Carlsbad, CA, USA) and Immobilon Western Chemiluminescent HRP Substrate (Millipore, Billerica, MA, USA), the blots were visualized using the ChemiDoc MP Imaging System (BioRad Laboratories, Hercules, CA, USA). Bands were quantified by densitometry using Image Lab software version 5.1 (BioRad Laboratories, Hercules, CA, USA).

2.6. Measurement of $\mathrm{NAD}^{+}$and $\mathrm{QA}$. The content of $\mathrm{NAD}^{+}$ and quinolinic acid (QA) in kidney tissue was examined using a rat $\mathrm{NAD}^{+}$ELISA kit and a rat QA ELISA kit (Jonln, Shanghai, China), respectively, following the manufacturer's protocol. In brief, the kidney cortex lysate was incubated with HRP-labeled detection antibody for $1 \mathrm{~h}$ at $37^{\circ} \mathrm{C}$. After washing 5 times with washing buffer, $50 \mu \mathrm{L}$ each of substrate $\mathrm{A}$ and $\mathrm{B}$ was added to each well and coincubated at $37^{\circ} \mathrm{C}$ for $15 \mathrm{~min}$ in the dark. Optical density (OD) values were measured using the BioTek microplate reader (BioTek Winooski, Vermont, USA) at $450 \mathrm{~nm}$ wavelength. The protein concentration of kidney cortex lysate was measured by the Bradford method. The values of $\mathrm{NAD}^{+}$and QA in the kidney were normalized to the protein concentrations.

2.7. Statistical Analysis. Data are expressed as mean\pm standard error of the mean (SEM). Data analysis was performed using GraphPad Prism 7.04 software (La Jolla, CA, USA). Comparisons were made using one-way ANOVA with Tukey's post hoc test. Statistical significance was defined as $P<0.05$.

\section{Results}

3.1. JPYSF Improved Renal Function in CKD Rats. Scr and BUN are the most commonly used clinical indicators to monitor renal function. The levels of Scr and BUN were significantly increased in both 5/6 Nx-induced CKD and adenine-induced CKD rats. After JPYSF treatment, Scr and BUN levels were dramatically reduced compared with the CKD group $(P<0.01)$ (Figure 2$)$.
3.2. JPYSF Attenuated Renal Tubulointerstitial Injury in CKD Rats. In PAS staining, massive tubular epithelial cells atrophy, partial renal tubular expansion, and loose tissue arrangement were observed in CKD rats, which could be improved by JPYSF treatment. Masson staining displayed that both $5 / 6 \mathrm{Nx}$ and adenine lead to an obvious accumulation of collagen fibrils (blue staining) in the renal interstitium. The fibrotic injury in CKD rats was markedly attenuated after JPYSF treatment (Figure 3).

3.3. JPYSF Decreased Renal Extracellular Matrix Deposition in CKD Rats. Renal fibrosis is a hallmark of CKD and is characterized by extracellular matrix deposition. We assessed FN, Col-IV, and $\alpha$-SMA expression by Western blot analysis in the kidney of CKD rats with or without JPYSF treatment. CKD rats had significantly increased FN, Col-IV, and $\alpha$-SMA expression compared with the sham or control group. However, JPYSF treatment markedly decreased these proteins expression in the kidney of CKD rats (Figure 4).

\subsection{JPYSF Promoted QPRT/NAD ${ }^{+} /$SIRT3 Signal in CKD Rats.} The level of $\mathrm{NAD}^{+}$in the kidney of CKD rat was significantly lower compared with normal rat (Figures 5(a) and 5(c)). On the contrary, the content of $\mathrm{QA}, \mathrm{NAD}^{+}$precursor in de novo biosynthesis, was elevated in the kidney of CKD rat (Figures 5(b) and 5(d)). Not surprisingly, the expression of the enzyme QPRT that catalyzes QA was downregulated in $\mathrm{CKD}$ rats. In addition, $\mathrm{NAD}^{+}$-dependent deacetylase SIRT3 was also downregulated in CKD rats (Figures 5(e)-5(h)). Administration of JPYSF markedly increased QPRT expression, lowered QA content, increased $\mathrm{NAD}^{+}$level, and upregulated SIRT3 expression in CKD rats. These results indicated that QPRT/NAD ${ }^{+} /$SIRT3 signal was suppressed in the kidney of CKD rats and could be promoted by JPYSF treatment.

3.5. JPYSF Modulated Mitochondrial Dynamics in CKD Rats. Mitochondrial dynamics was governed by the balance of mitochondrial fission and fusion. Drp- 1 and OPA- 1 are the key regulators of mitochondrial fission and fusion, respectively. Our data found that Drp-1 was upregulated and OPA1 was downregulated in CKD rats. JPYSF treatment could 


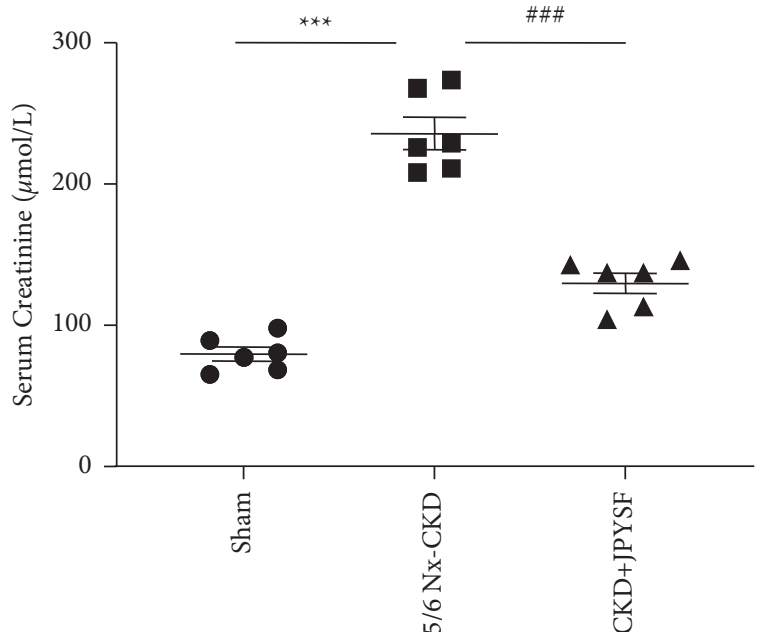

(a)

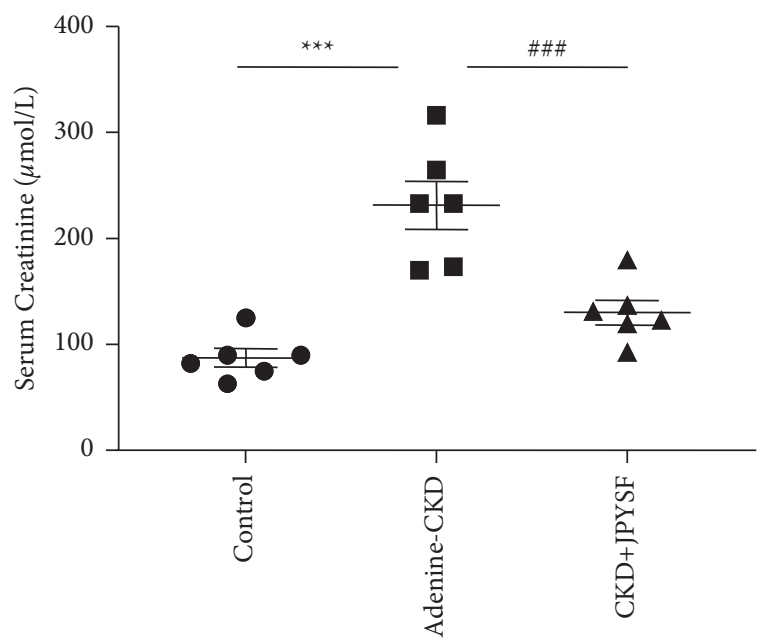

(c)

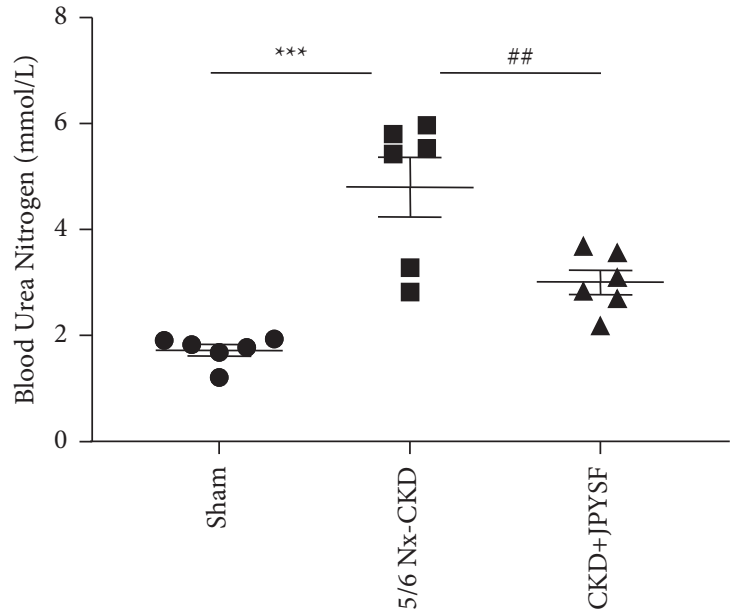

(b)

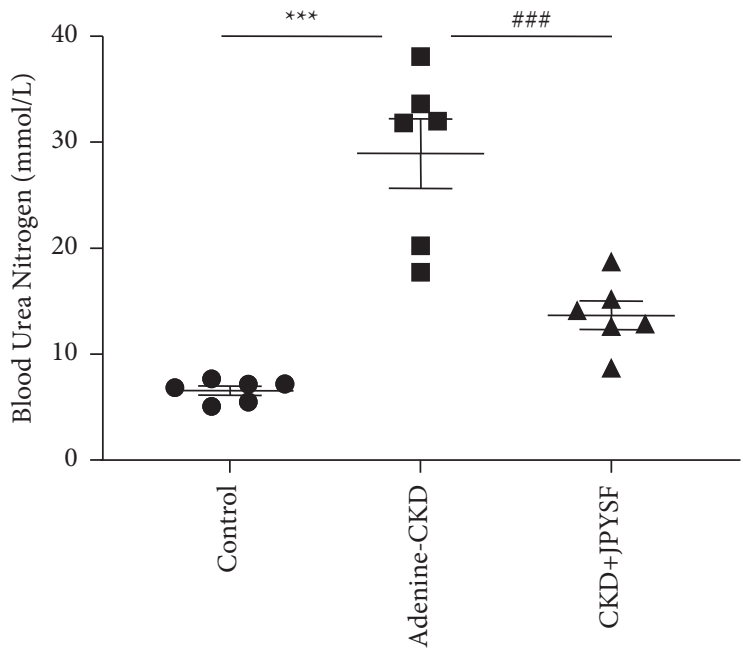

(d)

FIGURE 2: JPYSF improved kidney function in CKD rats. (a) The level of serum creatinine in each group in the setting of 5/6 Nx-induced CKD. (b) The level of blood urea nitrogen in each group in the setting of 5/6 Nx-induced CKD. (c) The level of serum creatinine in each group in the setting of adenine-induced CKD. (d) The level of blood urea nitrogen in each group in the setting of adenine-induced CKD. Data are presented as the means \pm SEM, $n=6$ rats per group $\left({ }^{* * *} P<0.001\right.$ compared with the sham or the control group; ${ }^{\# \#} P<0.01$ compared with the 5/6 Nx-CKD group; ${ }^{\# \#} P<0.001$ compared with the 5/6 Nx-CKD or the adenine-CKD group).
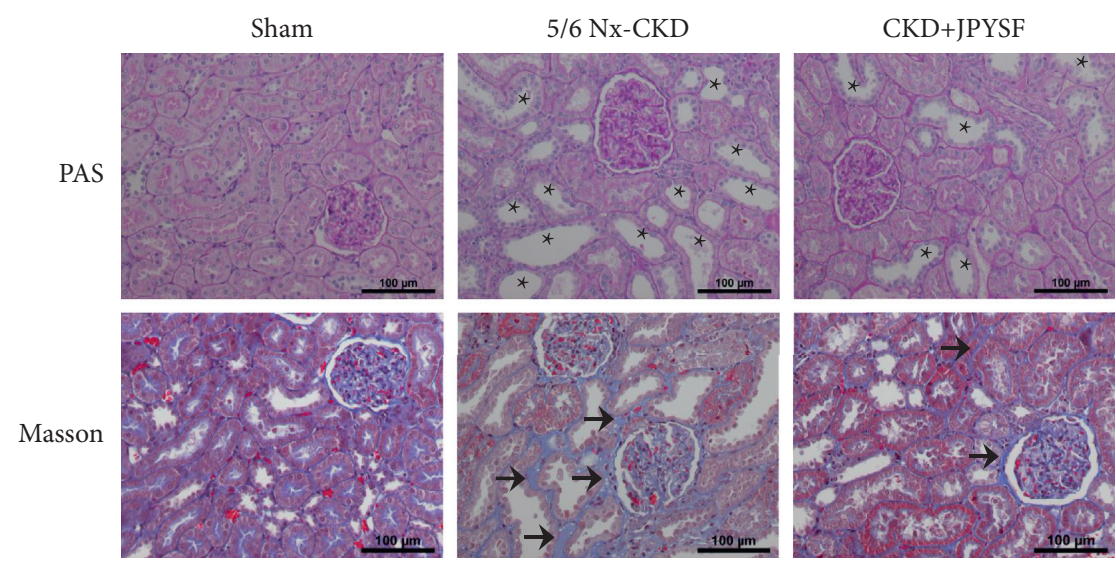

(a)

Figure 3: Continued. 

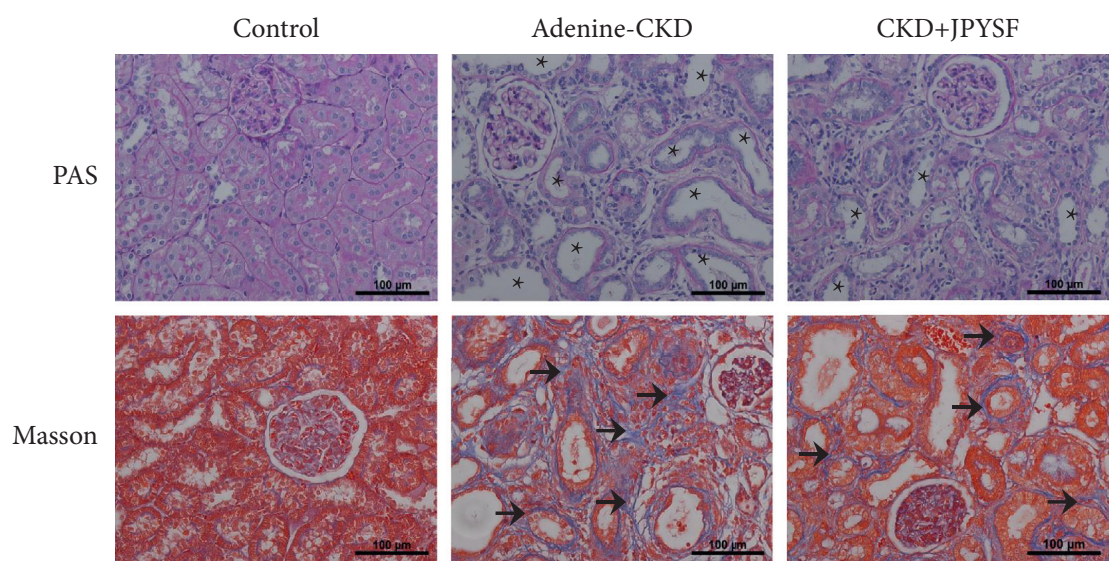

(b)

FIgURe 3: JPYSF ameliorated renal injury in CKD rats. (a) PAS and Masson staining in the setting of 5/6 Nx-induced CKD. (b) PAS and Masson staining in the setting of adenine-induced CKD. Asterisk indicates tubular expansion; arrow indicates collagen fibril. All images are shown at identical magnification, $\times 200$, scale bar $=100 \mu \mathrm{m}$.
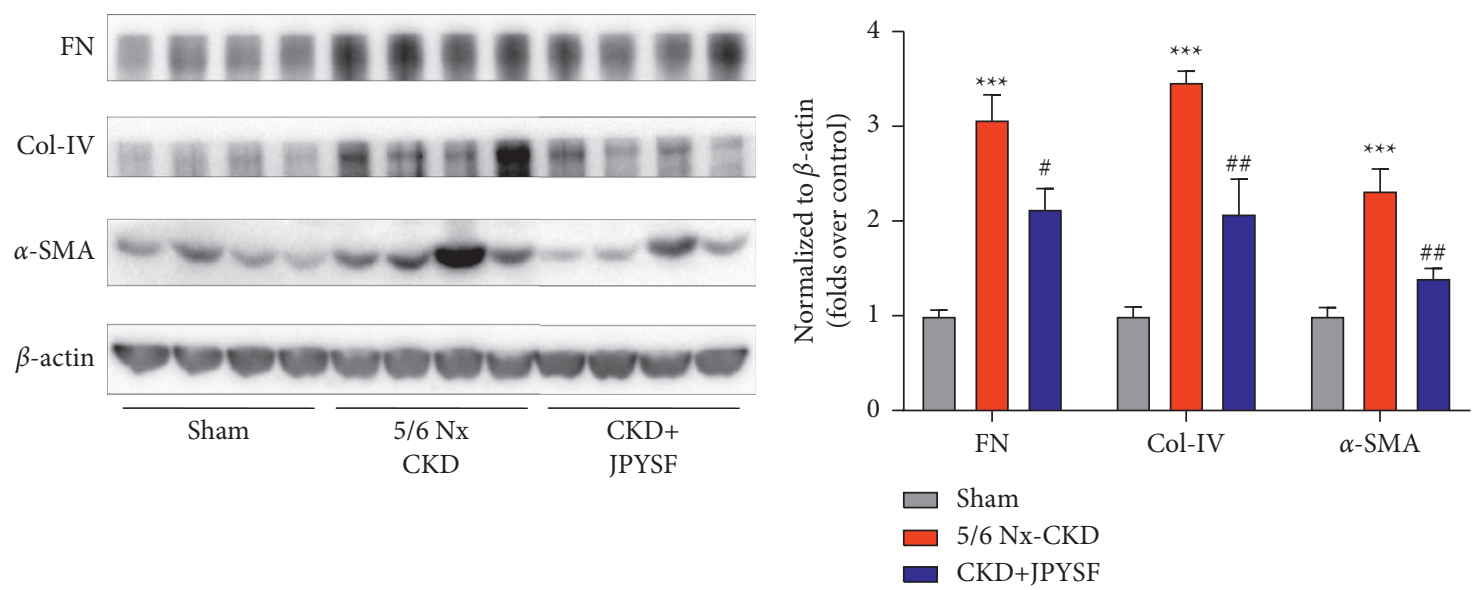

(a)
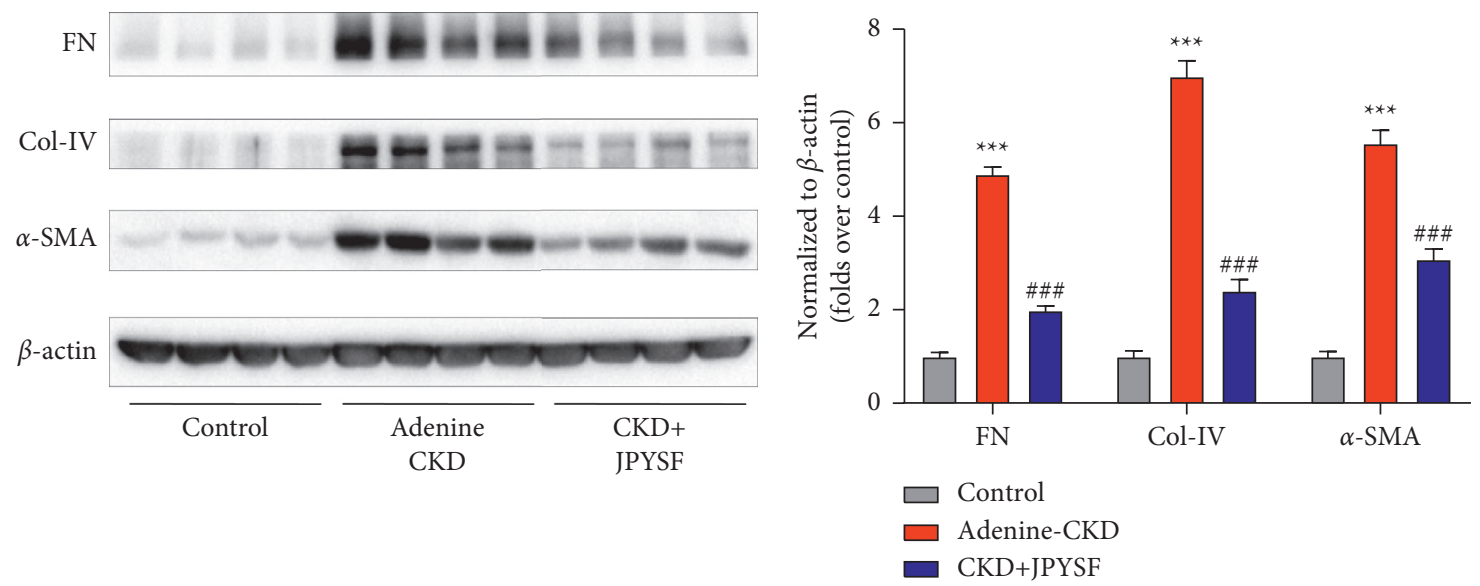

(c)

(d)

FIGURE 4: JPYSF suppressed the expression of fibrotic markers in the kidney of CKD rats. Representative Western blot images (a) and densitometric analyses (b) of FN, Col-IV, and $\alpha$-SMA protein expression in the setting of 5/6 Nx-induced CKD. Representative Western blot images (c) and densitometric analyses (d) of FN, Col-IV, and $\alpha$-SMA protein expression in the setting of adenine-induced CKD. All proteins expression was normalized to $\beta$-actin content. Data are presented as the means \pm SEM, $n=6$ rats per group $\left({ }^{* * *} P<0.001\right.$ compared with the sham or the control group; ${ }^{\#} P<0.05$ compared with the $5 / 6 \mathrm{Nx}$-CKD group; ${ }^{\#} P<0.01$ compared with the $5 / 6 \mathrm{Nx}-\mathrm{CKD}$ group; $\# \#$ $P<0.001$ compared with the adenine-CKD group). 


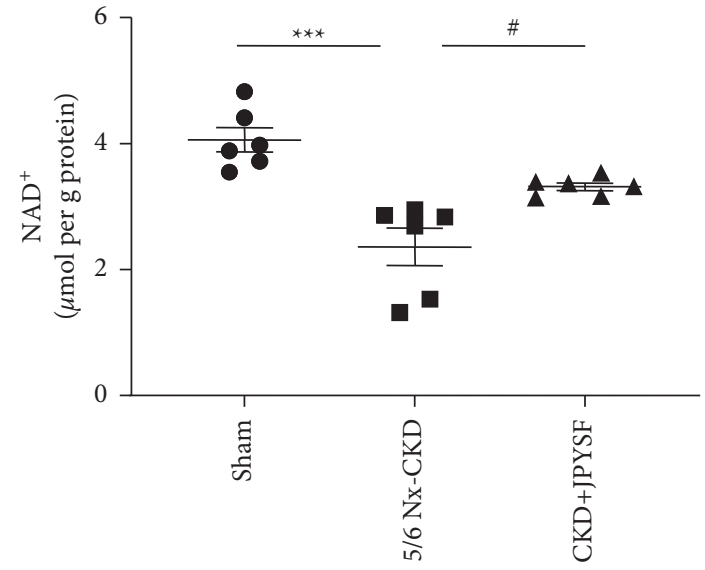

(a)

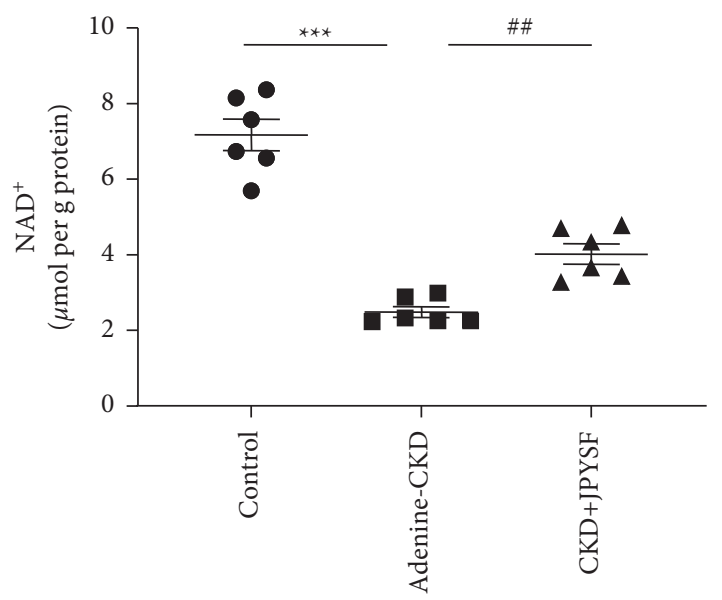

(c)
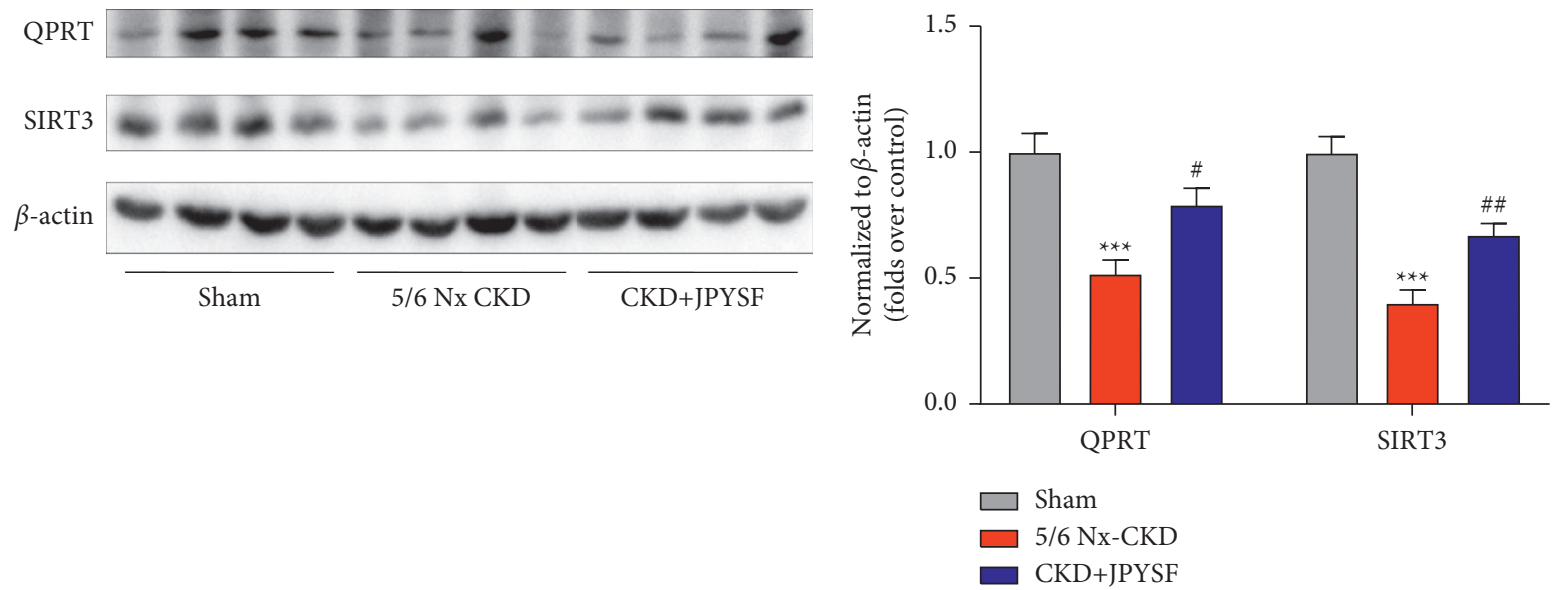

(e)

QPRT

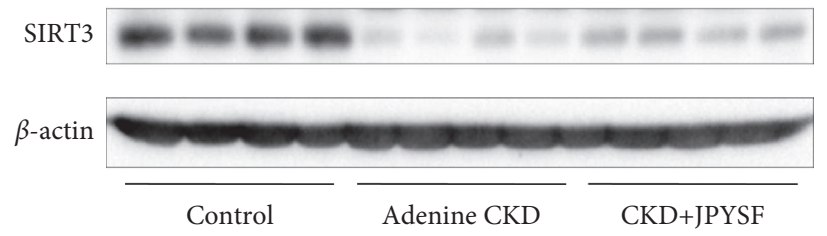

(g)

FIGURE 5: Continued. 


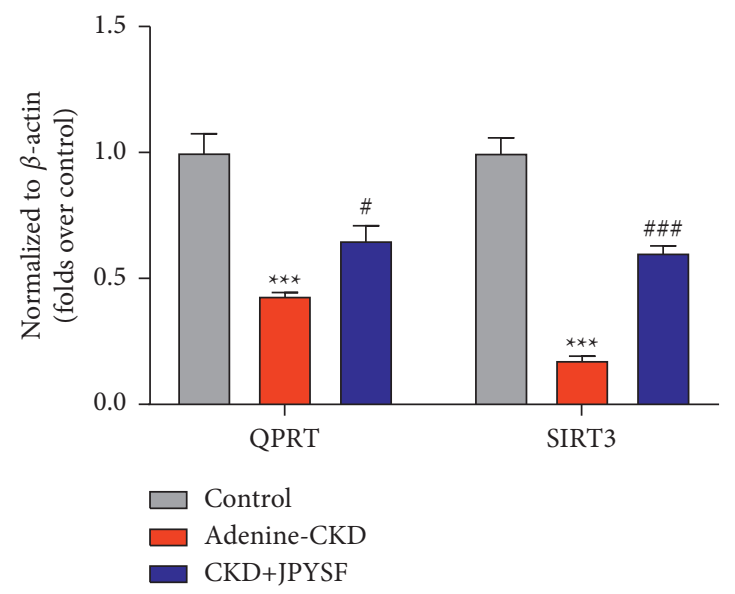

(h)

FIGURE 5: Effect of JPYSF on the QPRT/NAD ${ }^{+} /$SIRT3 pathway in the kidney of CKD rats. The levels of NAD ${ }^{+}$(a) and quinolinic acid (b) in each group in the setting of 5/6 Nx-induced CKD. The levels of $\mathrm{NAD}^{+}(\mathrm{c})$ and quinolinic acid (d) in each group in the setting of adenineinduced CKD. Representative Western blot images (e) and densitometric analyses (f) of QPRT and SIRT3 protein expression in the setting of 5/6-Nx induced CKD. Representative Western blot images ( $\mathrm{g}$ ) and densitometric analyses $(\mathrm{h})$ of QPRT and SIRT3 protein expression in the setting of adenine-induced CKD. All proteins expression was normalized to $\beta$-actin content. Data are presented as the means \pm SEM, $n=6$ rats per group $\left({ }^{* *} P<0.001\right.$ compared with the sham or the control group; ${ }^{\#} P<0.05$ compared with the $5 / 6 \mathrm{Nx}$-CKD or the adenineCKD group; ${ }^{\#} P<0.01$ compared with the $5 / 6 \mathrm{Nx}$-CKD or the adenine-CKD group; ${ }^{\# \#} P<0.001$ compared with the adenine-CKD group).

reverse the expression pattern of Drp-1 and OPA-1 (Figure 6). This result suggested that JPYSF could modulate mitochondrial dynamics in CKD rats.

\section{Discussion}

In the present study, two CKD rat models were successfully established by $5 / 6 \mathrm{Nx}$ and feeding with adenine-containing feed. Administration of JPYSF improved renal function and pathological injury and decreased renal extracellular matrix deposition in CKD rats. In addition, CKD rats exhibited suppressed QPRT/NAD ${ }^{+} / \mathrm{SIRT} 3$ signal, increased mitochondrial fission, and decreased mitochondrial fusion. JPYSF treatment promoted QPRT/NAD ${ }^{+} /$SIRT3 signal and restored mitochondrial fission/fusion balance.

TCM believes that the basic pathogenesis of CKD is deficiency of spleen and kidney Qi, dampness, blood stasis, and turbidity. JPYSF was founded based on this theory and has the effect of invigorating the spleen and kidney, promoting blood circulation, and removing turbidity. In order to obtain more solid evidence, we used two rat models to study the efficacy and mechanism of JPYSF in the treatment of CKD. Both 5/6 Nx and adenine feed can induce CKD phenotype [20-22]; the difference is that $5 / 6 \mathrm{Nx}$ requires surgery and a longer experimental period. In this study, these two CKD models were successfully established, and JPYSF could effectively improve CKD (Figures 2 and 3), which laid the foundation for mechanism research.

The unbalanced mitochondrial fission/fusion is consistent with our previous results $[8,9]$, but the regulatory mechanism is still unclear. Sirtuins are an evolutionarily conserved family comprising of seven members (SIRT1-SIRT7) in mammals, among which three (SIRT3, SIRT4, and SIRT5) are exclusively localized to mitochondria
[23]. Striking mitochondrial protein hyperacetylation was detected in SIRT3-deficient mice but not SIRT4 and SIRT5deficient mice, suggesting that SIRT3 is a major mitochondrial deacetylase [11]. SIRT3 has been documented as an important molecule in regulating mitochondrial function with the implications in metabolic enzyme activity, oxidative phosphorylation efficacy, and antioxidant machinery [24-26]. Emerging evidence indicates that SIRT3 is critical in sustaining mitochondrial dynamics. In the cisplatin-induced AKI mice model, renal SIRT3 was downregulated leading to increased expression of Drp-1, accompanied by a decrease in OPA-1, thus carrying mitochondria dynamics toward fission and fragmentation. Upregulation of SIRT3 resulted in mitochondrial fusion, limiting segregation and protection against cisplatin-induced AKI [12]. Specific activation of SIRT3 by honokiol was capable to protect vitiligo melanocytes against oxidative stress via the regulation of OPA-1-dependent mitochondrial dynamics [13]. SIRT3 could alleviate renal ischemia-reperfusion injury through enhancing mitochondrial fusion and activating the ERKOPA-1 signaling pathway [27]. Recently, SIRT3 was found to confer neuroprotection in Huntington's disease by regulation of mitochondrial dynamics [14]. Consistent with previous studies, our data show that SIRT3 was downregulated, accompanied by an increase in Drp-1 and decrease in OPA-1 in CKD rat models. However, the specific mechanism by which SIRT3 regulates mitochondrial dynamics needs further study.

As a $\mathrm{NAD}^{+}$-dependent deacetylase, the function of SIRT3 is regulated by levels of $\mathrm{NAD}^{+}$. Intracellular $\mathrm{NAD}^{+}$can be produced through either de novo synthesis from tryptophan or via salvage pathways from precursor molecules such as nicotinamide, nicotinic acid, or nicotinamide riboside [28]. Klimova et al. reported that $\mathrm{NAD}^{+}$precursor, nicotinamide 

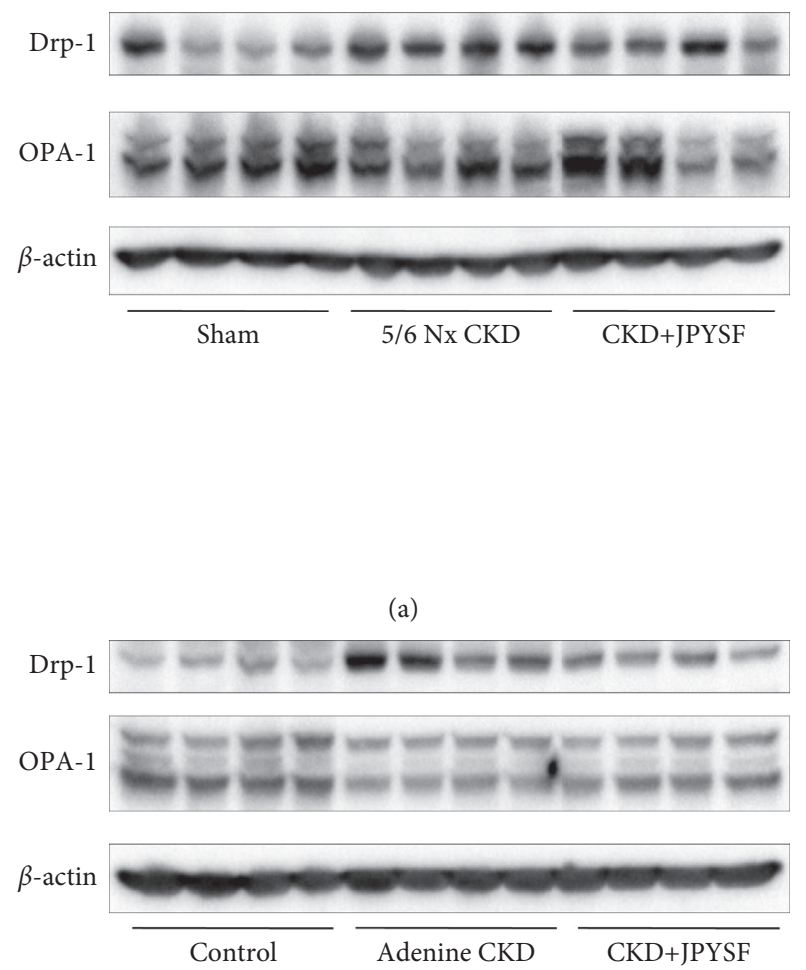

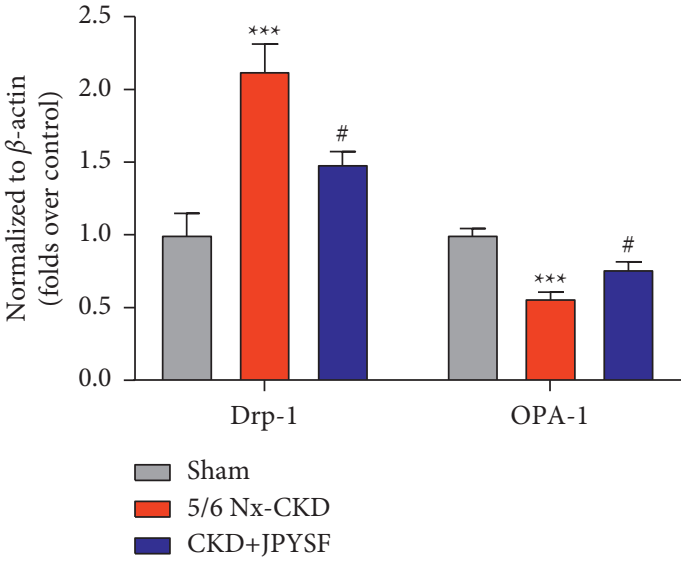

(b)

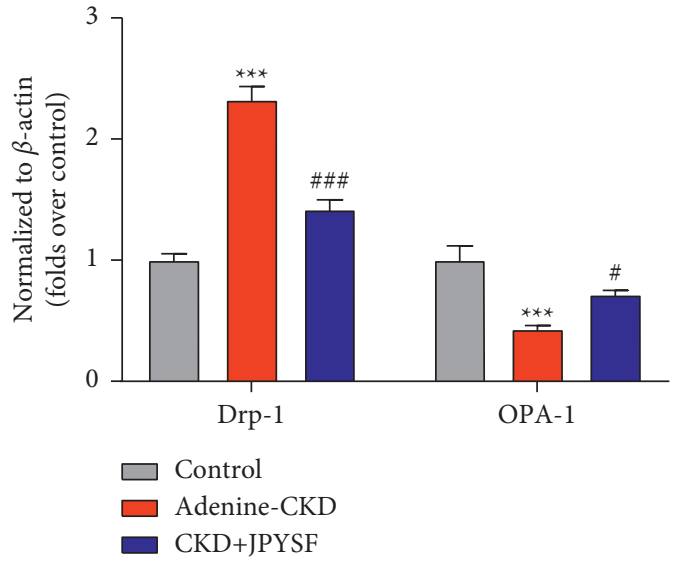

(d)

FIGURE 6: JPYSF modulated mitochondrial dynamics in CKD rats. Representative Western blot images (a) and densitometric analyses (b) of Drp-1 and OPA-1 protein expression in the setting of 5/6 Nx-induced CKD. Representative Western blot images (c) and densitometric analyses (d) of Drp-1 and OPA-1 protein expression in the setting of adenine-induced CKD. All proteins expression was normalized to $\beta$-actin content. Data are presented as the means \pm SEM, $n=6$ rats per group ( ${ }^{* * *} P<0.001$ compared with the sham or the control group; ${ }^{\#} P<0.05$ compared with the $5 / 6 \mathrm{Nx}$-CKD or the adenine-CKD group; ${ }^{\# \#} P<0.001$ compared with the adenine-CKD group).

mononucleotide, altered mitochondrial dynamics in the brain by reducing fragmentation of neuronal mitochondria by the SIRT3-dependent mechanism [29]. In recent years, the critical role of de novo $\mathrm{NAD}^{+}$synthesis in health and disease has been gradually revealed [15-17]. A well-designed study found that impairment of $\mathrm{NAD}^{+}$synthesis is a hallmark of AKI and CKD. However, nicotinamide riboside supplementation aimed to boost $\mathrm{NAD}^{+}$production via salvage pathways was beneficial in ischemic AKI but not in CKD models [30]. This study suggested that impaired de novo $\mathrm{NAD}^{+}$synthesis played more important role in CKD. In de novo $\mathrm{NAD}^{+}$synthesis, tryptophan and other upstream metabolites have multiple metabolic fates, making QA the first fully committed $\mathrm{NAD}^{+}$precursor in de novo biosynthesis. QPRT is the enzyme that connects the initial steps of opening QA's pyrrole ring to the final steps of $\mathrm{NAD}^{+}$biosynthesis [31]. Similar with a previous study in AKI, our data also found low expression of renal QPRT, QA accumulation, and low $\mathrm{NAD}^{+}$ levels in CKD models, which suggested that de novo $\mathrm{NAD}^{+}$synthesis was impaired in CKD and may represent therapeutic target for CKD treatment.

\section{Conclusions}

In conclusion, administration of JPYSF effectively alleviated CKD progression in two rat models, which may be related with regulation of the QPRT/NAD ${ }^{+} / \mathrm{SIRT} 3 /$ mitochondrial dynamics pathway.

\section{Abbreviations}

5/6 Nx: 5/6 Nephrectomy

$\alpha$-SMA: $\alpha$-Smooth muscle actin

AKI: Acute kidney injury

BUN: Blood urea nitrogen

CKD: Chronic kidney disease

Col-IV: Type IV collagen

Drp-1: Dynamin-related protein 1

FN: $\quad$ Fibronectin

JPYSF: Jian-Pi-Yi-Shen formula

MFN: Mitofusin

NAD: Nicotinamide adenine dinucleotide

OPA-1: Optic atrophy 1 
PAS: $\quad$ Periodic acid-Schiff

QA: Quinolinic acid

QPRT: Quinolinate phosphoribosyltransferase

SIRT3: Sirtuin 3

Scr: $\quad$ Serum creatinine

TCM: Traditional Chinese medicine.

\section{Data Availability}

The data used to support the findings of this study are available from the corresponding author upon request.

\section{Disclosure}

The funders have no role in the design of the study and collection, analysis, and interpretation of data, and in writing the manuscript.

\section{Conflicts of Interest}

The authors declare that there are no conflicts of interest.

\section{Acknowledgments}

This study was supported by the Natural Science Foundation of China (81973602 and 81804052), Shenzhen Science and Technology Program (JCYJ20190812161001790, JCYJ2021032 4111210029, JSGG20191129102216637, JCYJ2018030217370 8520, and JCYJ20180507183842516), and Natural Science Foundation of Guangdong Province (2020A1515011151 and 2018A030313305).

\section{References}

[1] G. B. D. Chronic Kidney Disease Collaboration, "Global, regional, and national burden of chronic kidney disease, 19902017: a systematic analysis for the Global Burden of Disease Study 2017," Lancet, vol. 395, no. 10225, pp. 709-733, 2020.

[2] E. F. Carney, "The impact of chronic kidney disease on global health," Nature Reviews Nephrology, vol. 16, no. 5, p. 251, 2020.

[3] C. F. Hsieh, S. L. Huang, C. L. Chen, W. T. Chen, H. C. Chang, and C. C. Yang, "Non-aristolochic acid prescribed Chinese herbal medicines and the risk of mortality in patients with chronic kidney disease: results from a population-based follow-up study," BMJ Open, vol. 4, no. 2, Article ID e004033, 2014.

[4] M.-Y. Lin, Y.-W. Chiu, J.-S. Chang et al., "Association of prescribed Chinese herbal medicine use with risk of end-stage renal disease in patients with chronic kidney disease," Kidney International, vol. 88, no. 6, pp. 1365-1373, 2015.

[5] C. F. Hsieh, H. C. Chang, S. L. Huang, C. L. Chen, W. T. Chen, and C. C. Yang, "Prescribed renoprotective Chinese herbal medicines were associated with a lower risk of all-cause and disease-specific mortality among patients with chronic kidney disease: a population-based follow-up study in taiwan," $E v-$ idence-Based Complementary and Alternative Medicine, vol. 2017, Article ID 5632195, 2017.

[6] X. Wang, S. Yu, Q. Jia et al., "NiaoDuQing granules relieve chronic kidney disease symptoms by decreasing renal fibrosis and anemia," Oncotarget, vol. 8, no. 34, pp. 55920-55937, 2017.
[7] X. Liu, B. Zhang, S. Huang et al., "Metabolomics analysis reveals the protection mechanism of huangqi-danshen decoction on adenine-induced chronic kidney disease in rats," Frontiers in Pharmacology, vol. 10, p. 992, 2019.

[8] X. Liu, S. Huang, F. Wang et al., "Huangqi-danshen decoction ameliorates adenine-induced chronic kidney disease by modulating mitochondrial dynamics," Evidence-Based Complementary and Alternative Medicine, vol. 2019, Article ID 9574045, 2019.

[9] X. Liu, J. Chen, X. Liu et al., "Jian-Pi-Yi-Shen Formula ameliorates chronic kidney disease: involvement of mitochondrial quality control network," BMC Complementary and Alternative Medicine, vol. 18, no. 1, p. 340, 2018.

[10] R. Sabouny and T. E. Shutt, "Reciprocal regulation of mitochondrial fission and fusion," Trends in Biochemical Sciences, vol. 45, no. 7, pp. 564-577, 2020.

[11] D. B. Lombard, F. W. Alt, H.-L. Cheng et al., "Mammalian Sir2 homolog SIRT3 regulates global mitochondrial lysine acetylation," Molecular and Cellular Biology, vol. 27, no. 24, pp. 8807-8814, 2007.

[12] M. Morigi, L. Perico, C. Rota et al., "Sirtuin 3-dependent mitochondrial dynamic improvements protect against acute kidney injury," Journal of Clinical Investigation, vol. 125, no. 2, pp. 715-726, 2015.

[13] X. Yi, W. Guo, Q. Shi et al., "SIRT3-Dependent mitochondrial dynamics remodeling contributes to oxidative stress-induced melanocyte degeneration in vitiligo," Theranostics, vol. 9, no. 6, pp. 1614-1633, 2019.

[14] L. Naia, C. Carmo, S. Campesan et al., "Mitochondrial SIRT3 confers neuroprotection in Huntington's disease by regulation of oxidative challenges and mitochondrial dynamics," Free Radical Biology and Medicine, vol. 163, 2020.

[15] E. Katsyuba, A. Mottis, M. Zietak et al., "De novo NAD+ synthesis enhances mitochondrial function and improves health," Nature, vol. 563, no. 7731, pp. 354-359, 2018.

[16] P. S. Minhas, L. Liu, P. K. Moon et al., "Macrophage de novo $\mathrm{NAD}+$ synthesis specifies immune function in aging and inflammation," Nature Immunology, vol. 20, no. 1, pp. 50-63, 2019.

[17] A. Poyan Mehr, M. T. Tran, K. M. Ralto et al., "De novo $\mathrm{NAD}+$ biosynthetic impairment in acute kidney injury in humans," Nature Medicine, vol. 24, no. 9, pp. 1351-1359, 2018.

[18] F. Wang, S. Huang, Q. Chen et al., "Chemical characterisation and quantification of the major constituents in the Chinese herbal formula Jian-Pi-Yi-Shen pill by UPLC-Q-TOF-MS/MS and HPLC-QQQ-MS/MS," Phytochemical Analysis, vol. 31, 2020.

[19] X. Liu, D. Luo, S. Huang et al., "Impaired nicotinamide adenine dinucleotide biosynthesis in the kidney of chronic kidney disease," Frontiers in Physiology, vol. 12, Article ID 723690, 2021.

[20] Y. Han, J. S. Lu, Y. Xu, L. Zhang, and B. F. Hong, "Rutin ameliorates renal fibrosis and proteinuria in 5/6-nephrectomized rats by anti-oxidation and inhibiting activation of TGF $\beta 1$-smad signaling," International Journal of Clinical and Experimental Pathology, vol. 8, no. 5, pp. 4725-4734, 2015.

[21] H. Askari, B. Seifi, and M. Kadkhodaee, "Evaluation of renalhepatic functional indices and blood pressure based on the progress of time in a rat model of chronic kidney disease," Nephro-Urology Monthly, vol. 8, no. 3, Article ID e37840, 2016.

[22] T. Yokozawa, P. D. Zheng, H. Oura, and F. Koizumi, "Animal model of adenine-induced chronic renal failure in rats," Nephron, vol. 44, no. 3, pp. 230-234, 1986. 
[23] W. C. Hallows, B. C. Smith, S. Lee, and J. M. Denu, "Ure(k)a! Sirtuins regulate mitochondria," Cell, vol. 137, no. 3, pp. 404-406, 2009.

[24] W. Yang, K. Nagasawa, C. Münch et al., "Mitochondrial sirtuin network reveals dynamic SIRT3-dependent deacetylation in response to membrane depolarization," Cell, vol. 167, no. 4, pp. 985-1000, 2016.

[25] O. Ozden, S.-H. Park, B. A. Wagner et al., "SIRT3 deacetylates and increases pyruvate dehydrogenase activity in cancer cells," Free Radical Biology and Medicine, vol. 76, pp. 163-172, 2014.

[26] X. Qiu, K. Brown, M. D. Hirschey, E. Verdin, and D. Chen, "Calorie restriction reduces oxidative stress by SIRT3-mediated SOD2 activation," Cell Metabolism, vol. 12, no. 6, pp. 662-667, 2010.

[27] Q. Wang, J. Xu, X. Li et al., "Sirt3 modulate renal ischemiareperfusion injury through enhancing mitochondrial fusion and activating the ERK-OPA1 signaling pathway," Journal of Cellular Physiology, vol. 234, no. 12, pp. 23495-23506, 2019.

[28] E. Katsyuba and J. Auwerx, "Modulating NAD + metabolism, from bench to bedside," The EMBO Journal, vol. 36, no. 18, pp. 2670-2683, 2017.

[29] N. Klimova, A. Long, and T. Kristian, "Nicotinamide mononucleotide alters mitochondrial dynamics by SIRT3dependent mechanism in male mice," Journal of Neuroscience Research, vol. 97, no. 8, pp. 975-990, 2019.

[30] A. Faivre, E. Katsyuba, T. Verissimo et al., "Differential role of nicotinamide adenine dinucleotide deficiency in acute and chronic kidney disease," Nephrology Dialysis Transplantation, vol. 36, 2020.

[31] K. Yaku, K. Okabe, and T. Nakagawa, "NAD metabolism: implications in aging and longevity," Ageing Research Reviews, vol. 47, pp. 1-17, 2018. 